\title{
Genetic Structure and Phylogenetic Status of Rice Brown Plant Hopper (BPH), Nilaparvata lugens Isolated from Kerala, India
}

\author{
K. Mashhoor ${ }^{1}$, K.V. Lazar ${ }^{2}$, S. Shanas ${ }^{3}$, N. Ramesh ${ }^{4} *$ \\ ${ }^{1}$ Department of Biotechnology, E.M.E.A. College of Arts and Science, Kondotti, Malappuram, Kerala, 673638, India. \\ ${ }^{2}$ Department of Environmental Science, Central University of Kerala, Nileswaram, Kasaragod, Kerala, 671314, India. \\ ${ }^{3}$ Division of Entomology, Rice ResearchStation, Kerala Agricultural University, Thekkekara, Moncompu, Alappuzha, Kerala, 688503, India. \\ ${ }^{4}$ Department of Biotechnology, J.J. College of Arts and Science, Namanasamudram, Pudukkottai, Tamilnadu, 622404, India.
}

\section{ARTICLE INFO \\ Article history: \\ Received on: 08/08/2015 \\ Revised on: 07/09/2015 \\ Accepted on: 26/09/2015 \\ Available online: 22/10/2015}

Key words:

Cytochrome oxidase, $N$.

lugens, Phylogeny, Genetic

divergence.

\begin{abstract}
Brown plant hopper, Nilaparvata lugens is a major pest of paddy, which damages the plants directly by sucking the sap and indirectly by transmission of rice viruses such as ragged stunt virus and grassy stunt virus. Geographically N. lugens are distributed throughout South and South East Asia where rice is grown and feed the phloem of plant. Due to the uncontrolled use of insecticides, insecticide resistant plant hoppers were emerged in many geographical locations like Japan, South and Southeast Asia etc. In this study we have described the Phylogenetic status of $N$. lugens isolated from Kerala, India. This study revealed that, N. lugens isolated from Andhra Pradesh and a few populations of $N$. lugens isolated from China and Japan were genetically similar to $N$. lugens isolated from Kerala. The result of this study also revealed the biasness of COI sequence of $N$. lugens to nucleotides AT.
\end{abstract}

\section{INTRODUCTION}

Brown plant hopper (BPH), Nilaparvata lugens is one of the most major pest of paddy, which cause huge destruction of rice plants in many rice producing countries [1]. BPH damages plants directly by sucking the sap and indirectly by transmission of rice viruses such as ragged stunt virus and grassy stunt virus $[2,3]$. BPH is known to occur in Asia since late forties, it was earlier only a minor pest of rice [4] and in the 1970s and early 1980 s the pest poses tremendous problems and was referred to as "a threat to Asian rice production" [5]. N. lugens distributed throughout South and South East Asia where rice is grown and they feed the phloem of plant. It infests the rice crop at all stages of plant growth. Both nymph and adult suck the sap from the base of the tillers, plants turn yellow and dry up rapidly. Round yellow patches appear in the paddy field in the early stages of infestation, which soon turn brownish due to the drying up of the plants and this condition is called 'hopperburn'. Severe infestation causes lodging of the crop resulting in yield loss ranging from $10-70 \%$ [6]. Plant hoppers have two distinct winged forms: macropterous and brachypterous. Macropterous are long-winged and brachypterous are short-winged and they are ochraceous brown dorsally and brown ventrally. The females lacerate the midrib of the leaf blade or the leaf sheath to lay egg

* Corresponding Author

Department of Biotechnology, E.M.E.A. College of Arts and Science,

Kondotti, Malappuram, Kerala, 673638, India.

Email:mashhoork@gmail.com masses in the parenchymatous tissue. The average member of eggs laid varies from $250-350$. The incubation period is $6-9$ days and the nymphal period is $10-18$ days. They undergo four to five molts. The total life-cycle occupies 16 to 27 days $[6,7]$. In the chemical control of BPH, carbamates have a generally higher level of ovicidal activity than the organophosphates. Insecticide-induced resurgence of $N$. lugens has been reported from many tropical countries. Destruction of the natural enemies is generally recognized as the primary cause of Insecticide-induced resurgence [7]. Extensive use of chemical insecticides for the control of BPH on rice can cause serious problems including toxicity to natural enemies of BPH such as Anagrus nilaparvatae [8], increased production cost, and possible long term agro-ecosystem and human health damage $[9,10]$. For the control of $N$. lugens, insecticide granules are less effective than sprays or dusts, particularly when applied to older plants with a greater biomass. Insecticide resistant plant hoppers have been most common in Japan where the insecticide use rate on rice is much higher than in other tropical countries. $N$. lugens has also developed resistance to carbofuran in South and Southeast Asia [7].

Different biotypes and sympatric biological species of $N$. lugens have been reported in many studies [11, 12]. Mun et al. [13] analyzed the genetic variation among Asian populations of rice plant hoppers, $N$. lugens and Sogatella furcifera (Hemiptera: Delphacidae) using mitochondrial DNA sequences. The analyses of isozymes and RAPD-PCR markers indicated that BPH captured from rice plant has high esterase activity compared to BPH captured from $L$. hexandra and they represent two distinct closely 
related sibling species in Malaysia [14, 15]. Latif et al. [16] reported that Direct amplified length polymorphism (DALP) also useful for the analysis of genetic polymorphisms in brown plant hopper, $N$. lugens populations. Recently 30 polymorphic microsatellite markers were developed from $N$. lugens genomic libraries using the method of Fast Isolation by AFLP of Sequence Containing Repeats [17]. Zang et al. [18] reported complete mitochondrial genome of $N$. lugens. Here we report the genetic variations of brown plant hopper, $N$. lugens isolated from Kerala.

\section{MATERIALS AND METHODS}

Adult of $N$. lugens were collected from the rice field of Kerala, India and the genomic DNA was isolated using GeNei Ultrapure Mammalian Genomic DNA Prep Kit as per the Manufacturer's instruction. The 5' end of the mitochondrial cytochrome oxidase subunit I (COI) gene was amplified using the forward primer with DNA sequence 5' TCGAATTGAATTAGCACAACCAGG 3' and the reverse primer with DNA sequence of 5' AGCTCCTGCTAATACAGGTAAAGAT 3'.

The $25 \mu \mathrm{l}$ PCR reaction mixture consisted of 2 nanogram of genomic DNA $(1 \mu \mathrm{l}), 1 \mu \mathrm{l}$ each forward and reverse primers at a concentration of $10 \mu \mathrm{M}, 2.5 \mu \mathrm{l}$ of dNTPs $(2 \mathrm{mM}), 2.5 \mu \mathrm{l} 10 \mathrm{X}$ reaction buffer, $0.20 \mu \mathrm{l}$ Taq polymerase $(5 \mathrm{U} / \mu \mathrm{l})$ and $16.8 \mu \mathrm{l} \mathrm{H}_{2} \mathrm{O}$. The PCR profile consisted of an initial denaturation step of $3 \mathrm{~min}$ at $95{ }^{\circ} \mathrm{C}$, followed by 30 cycles of $10 \mathrm{sec}$ at $95^{\circ} \mathrm{C}, 45 \mathrm{sec}$ at $45^{\circ} \mathrm{C}$ and $45 \mathrm{sec}$ at $72{ }^{\circ} \mathrm{C}$ and ending with a final phase of $72{ }^{\circ} \mathrm{C}$ for 3 min. The PCR product was column purified using Mo Bio UltraClean PCR Clean-up Kit (Mo Bio Laboratories, Inc. California) as per the manufacturer's instructions. The purified PCR product was sequenced from both ends using the forward and reverse primers using the Sanger's sequencing method at SciGenom Laboratories Ltd., Cochin.

The forward and reverse sequences were assembled by using ClustalW (http://www.ebi.ac.uk/Tools/msa/clustalw2) after removing the forward and reverse primers and the consensus was taken for the analysis. The phylogenetic analysis was done using MEGA5 software.

\section{RESULTS AND DISCUSSION}

Brown plant hopper, $N$. lugens under the family Delphacidae is a one of the most destructive rice pest of Kerala and its attack is more common in Kerala. They damage plants by sucking the sap and also transmit viral diseases in plants. The adult brown plant hoppers are dimorphic. They were found in the field as mixed population of adult and nymph. The adult brown plant hoppers are may be winged or half winged. Both adult and nymph cause damage by sucking sap from the plant. The newly hatched nymphs are cottony white and turn to purple brown. The brown hopper populations multiply very fast and can migrate long distance and cause wide spread infestation. In the early stage of infestation yellowish patches were appeared and turns to brownish due to the drying of the plant. In the severely infested field, the rice gives a burned appearance (hopper burn). The partial coding sequence of mitochondrial COI gene of Nilaparavata lugens was PCR amplified using the forward primer with DNA sequence of 5' TCGAATTGAATTAGCACAACCAGG 3' and the reverse primer with DNA sequence of 5' AGCTCCTGCTAATACAGGTAAAGAT 3'. The PCR amplification of partial mitochondrial COI gene of $N$. lugens yielded a single product with about $550 \mathrm{bp}$ in size.

Table 1: Percentage of genetic divergence of the $N$. lugens isolated from Kerala and in the related species of order Hemiptera. The GenBank accession numbers are given in parenthesis.

\begin{tabular}{|c|c|}
\hline Name of species & \% of divergence \\
\hline Nilaparvata lugens (KC858992) & $0.00 \%$ \\
\hline Nilaparvata lugens (KC476395) & $0.00 \%$ \\
\hline Nilaparvata lugens (KC476394) & $0.00 \%$ \\
\hline Nilaparvata lugens (AB572318) & $0.00 \%$ \\
\hline Nilaparvata lugens (AB572314) & $0.00 \%$ \\
\hline Nilaparvata lugens (AB572300) & $0.00 \%$ \\
\hline Nilaparvata lugens (JN391181) & $0.00 \%$ \\
\hline Nilaparvata lugens (AB572309) & $0.55 \%$ \\
\hline Nilaparvata lugens (AB572308) & $0.55 \%$ \\
\hline Nilaparvata lugens (AB572307) & $0.55 \%$ \\
\hline Nilaparvata lugens (AB572306) & $0.55 \%$ \\
\hline Nilaparvata lugens (AB572305) & $0.55 \%$ \\
\hline Delphacidae sp. (HF968662) & $20.45 \%$ \\
\hline Trypetimorpha (KF298420) & $20.63 \%$ \\
\hline Trypetimorpha japonica (KF298420) & $20.63 \%$ \\
\hline Ommatissus lofouensis (KF298417) & $21.02 \%$ \\
\hline Sogatella furcifera $(\mathrm{KC} 476386)$ & $21.08 \%$ \\
\hline Tambinia sp. (JQ410451) & $22.67 \%$ \\
\hline Eusarima sp. (HM452253) & $22.71 \%$ \\
\hline Kallitaxila sinica (KF298415) & $22.93 \%$ \\
\hline Gergithus parallelus (HM452251) & $23.35 \%$ \\
\hline Hemisphaerius trilobulus (HM052840) & $23.35 \%$ \\
\hline Gergithus iguchii (HM452252) & $24.07 \%$ \\
\hline Mongoliana serrata (HM052830) & $24.09 \%$ \\
\hline Laodelphax striatellus (HM160143) & $25.47 \%$ \\
\hline Nesosydne chambersi (JQ771119) & $25.63 \%$ \\
\hline Gergithus sp. (HM052836) & $26.49 \%$ \\
\hline Oliarus polyphemus (HF674828) & $27.25 \%$ \\
\hline Lycorma delicatula (EU909203) & $29.85 \%$ \\
\hline Issidae sp. (HF968665) & $31.18 \%$ \\
\hline Catullia subtestacea (KF298414) & $31.87 \%$ \\
\hline Nephotettix virescens (HM160144) & $42.82 \%$ \\
\hline Nephotettix cincticeps (JN391184) & $42.82 \%$ \\
\hline
\end{tabular}

The sequence obtained after removing the primers used for PCR amplification was submitted to GenBank of NCBI (Accession: KF836703). The COI nucleotide sequence analysis revealed the composition of nucleotides in the COI gene of $N$. lugens isolated from Kerala. The COI sequence of $N$. lugens showed bias to nucleotide AT, with following composition of nucleotides $\mathbf{T}=35.10 \%, \mathbf{A}=31.10, \mathbf{C}=21.30 \%$ and $\mathbf{G}=12.50 \%$. The $N$. lugens isolated from Kerala showed similarity in the total nucleotide compositions and composition of nucleotides in the each position of codons with that of $N$. lugens isolated from Andhra Pradesh. Some populations of $N$. lugens isolated from China and Japan showed similarity and others showed variations in the total nucleotide composition and composition of nucleotides in the each position of codons with that of $N$. lugens isolated from Kerala. The genetic divergence analysis depicts the degree of divergence of different geographically isolated populations of $N$. lugens with $N$. lugens isolated from Kerala. N. lugens isolated from Andhra Pradesh (GenBank Accession No. KC858992) showed $0 \%$ divergence with $N$. lugens isolated from Kerala. 


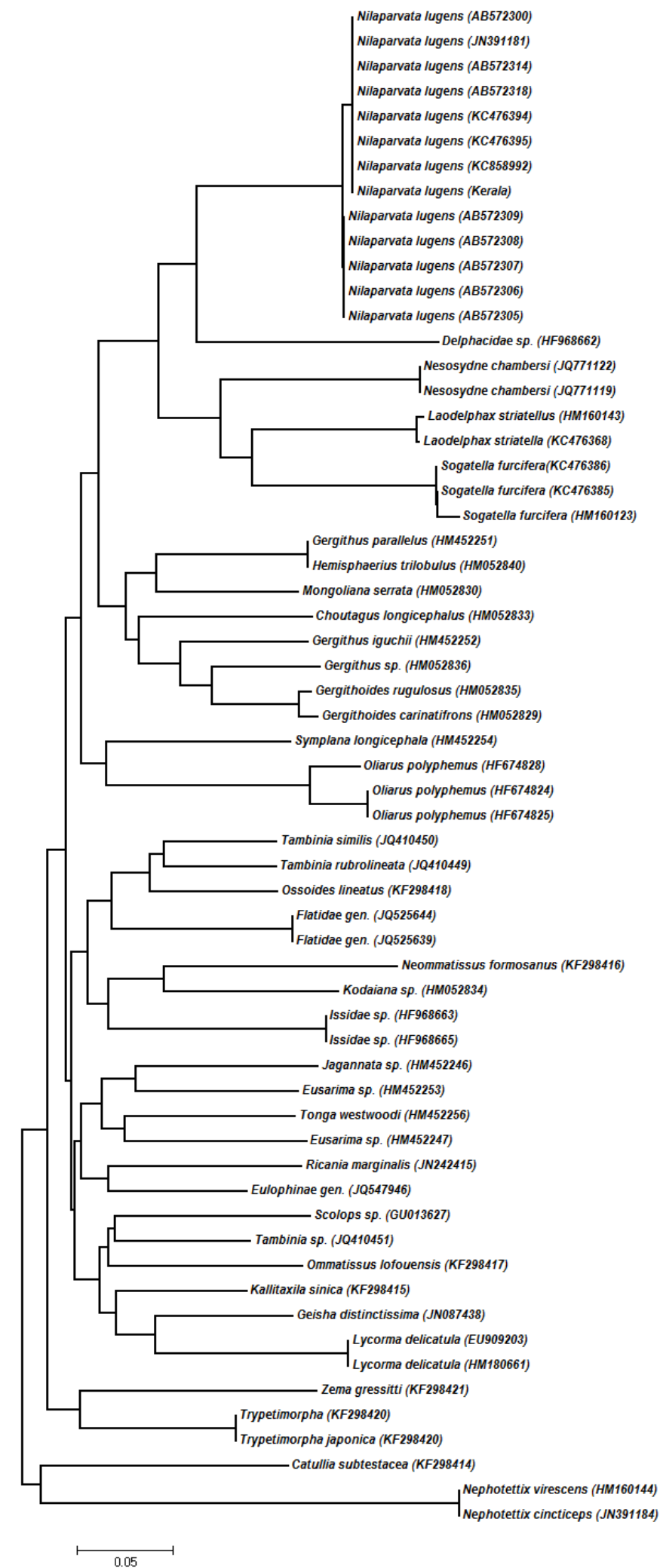

Fig. 1: Phylogenic relationship of $N$. lugens isolated from Kerala inferred by NJ tree method of MEGA5 software. The GenBank accession numbers are given in parenthesis.

Some populations of $N$. lugens isolated from China and Japan (GenBank Accession Nos. AB572318, AB572317, KC476395, KC476394 and B572300) showed 0\% divergence and others (GenBank Accession Nos. AB572309, AB572308, AB572307, JX880069 and JN391181) showed 0.55\% divergence with $N$. lugens isolated from Kerala (Table - 1). The phylogeny tree generated using Neighbour Joining method revealed the phylogenetic position of $N$. lugens isolated from Kerala (Fig. 1). The $N$. lugens isolated from different geographical location were aligned in single clad and divided in to two separate clad. Phylogeneticaly $N$. lugens isolated from Kerala is the nearest relative of $N$. lugens isolated from Andhra Pradesh and some populations of $N$. lugens isolated China and Japan, they together form a sub-clad.

The composition of nucleotides in the mtDNA $\mathrm{J}$ strand of $N$. lugens is $\mathbf{A}=41.96 \%, \mathbf{T}=34.99 \%, \mathbf{G}=9.43 \%$ and $\mathbf{C}=$ $13.62 \%$. The most of the heteropteran species mitogenomes nucleotide compositions are significantly biased toward $\mathbf{A}$ and $\mathbf{T}$. The mitochondrial genome of hemipteran species has considerable variation in base composition among different hemipteran species $[18,19]$. The COI nucleotide sequence analysis of $N$. lugens isolated from Kerala and other geographically isolated population also showed $68 \%$ AT content in the COI sequence which is varied from the total AT content of J strand. The related species of hemiptera also showed the same pattern result. These results indicated that, in the hemiptera the AT content of COI gene will be less compared to the total AT content of $\mathrm{J}$ strand. In the nucleotide triplet code, there is strong compulsion in the nucleotide changes in second position of all codons and first position of many codons. Due to the degenerative character of the triplet code third position of many codons and first position of some codons is less constraint. The variations in the strong constraint positions lead to the variations in the amino acids sequence. But the variations in the less constraint position will not affect (silent) the phenotype and these less constrained codon positions evolved at high rate [20,21]. In the case of COI sequence of $N$. lugens isolated from Kerala showed variation in the composition of nucleotides in the second position of codons with some populations identified from Japan and China (GenBank Accession Nos. AB572309, AB572308, AB572307, JX880069 and JN391181). These varied populations of $N$. lugens may be a different subspecies. The order Hemiptera is the largest group of the hemimetabolous insects [22]. The phylogenetic relationships of this order at the super family and suborder levels are still controversial [23, 24, 25]. The genetic divergence analysis revealed the genetic difference within the species $N$. lugens and between the related species of order Hemiptera. The COI sequence of $N$. lugens showed considerable variations with the related species. Therefore the COI sequence isolated in this study can be used as a barcode to identify this insect species.

\section{CONCLUSIONS}

The COI sequence of $N$. lugens exhibited considerable variation with other species. Therefore it can be used as DNA barcode to identify this organism. The $N$. lugens isolated from 
Andhra Pradesh and a few populations of $N$. lugens isolated from China and Japan were genetically similar to $N$. lugens isolated from Kerala. This study also concluded that the COI sequence of $N$. lugens has biasness to nucleotides AT.

\section{REFERENCES}

1. Bentur JS, Viraktamath BC. Rice planthoppers strike back. Current Science. 2008; 95: 441-443.

2. Velusamy R, Heinrichs EA. Electronic monitoring of feeding behavior of Nilaparvata lugens Homoptera: Delphacidae on resistant and susceptible rice cultivars. Environmental Entomology. 1986; 15: 678682.

3. Khush GS, Brar DS. Genetics of resistance to insects in crop plants. Advances in Agronomy. 1991; 45: 223-274.

4. Diwakar, MC. Genesis of rice brown plant hopper resurgence and spread in India-an overview, Plant Protection Bulletin. 1998; 50: 1-4.

5. International rice Research Institute. Brown plant hopper threat to rice production in Asia, Los Banos, Phillipines. 1979

6. Paul AV.N. Insect Pests and their management, Division of Entomology, Indian Agricultural Research, Institute, New Delhi110012. 2007.

7. Pathak MD and Khan ZR Insect Pest of Rice. International Rice Research Institute, Manila, Philippines. 1994

8. Wang HY, Yang Y, Su JY, Shen JL, Gao CF. Assessment of the impact of insecticides on Anagrus nilaparvatae Pang et Wang Hymenoptera: Mymanidae, an egg parasitoid of the rice planthopper, Nilaparvata lugens Hemiptera : Delphacidae. Crop Protection. 2008; 27: 514-522.

9. Huang J, Qiao F, Zhang L, Rozelle S. Farm pesticide, rice production and human health, Centre for Chinese Agricultural Policy, Chinese Academy of Sciences. Beijing, China. 2000.

10. Rola AC, Pingali, L P. Pesticides, Rice Productivity, and Farmers' Health: An Economic Assessment. Baños Los, Philippines, eds. and Washington D.C: International Rice Research Institute and World Resource Institute. 1993.

11. Claridge M F, DenHollander J and Morgan J C. "The status of weedassociated populations of the brown planthopper, Nilaparvata lugens Stal-host race or biological species?" Zoological Journal of the Linnean Society. 1985; 84: 77-90

12. Yang H, Ren X, Weng Q, Zhu L and He G. Molecular mapping and genetic analysis of a rice brown planthopper Nilaparvata lugens Stal resistance gene. Hereditas. 2002; 136: 39-43.

13. Mun JH, Song YH, Heong, KL and Roderick GK. Genetic variation among Asian populations of rice planthoppers, Nilaparvata lugens and Sogatella furcifera Hemiptera: Delphacidae: mitochondrial DNA sequences, Bull. Entomol. Res. 1999; 89, 245-253

14. Latif M A, Soon Guan T, Mohd YO. and Siraj, S. S. Evidence of sibling species in the brown planthopper complex Nilaparvata lugens detected fromshort and long primer random amplified polymorphic DNA ingerprints, Biochemical Genetics. 2008; 46: 520-537.
15. Latif M., Oma, MY, Tan, SG, Siraj SS and Ismail AR. Interpopulation crosses, inheritance study, and genetic variability in the brown planthopper complex, Nilaparvata lugens Homoptera: Delphacidae, Biochemical Genetics, 2010; 48: 266-286.

16. Latif MA, Rafii MY, Mazid MS, Ali ME, Ahmed F, Omar MY. and Tan SG. Genetic Dissection of Sympatric Populations of Brown Planthopper, Nilaparvata lugens $\mathrm{St}^{\circ}$ al, Using DALP-PCR Molecular Markers, The Scientific World Journal. 2012; Article ID 586831

17. Jing S, Zhou X, Yu H, Liu B, Zhang C, Wang S, Peng X, Zhu L, Ding $\mathrm{Y}$ and $\mathrm{He} \mathrm{G}$. Isolation and Characterization of Microsatellite Markers in Brown Planthopper Nilaparvata lugens Stål, Int. J. Mol. Sci. 2012. 13: 9527-9533

18. Zhang KJ, Zhu WC, Rong X, Zhang YK, Ding XL, Liu J, Chen DS, $\mathrm{Du} \mathrm{Y}$ and Hong XY. The complete mitochondrial genomes of two rice planthoppers, Nilaparvata lugens and Laodelphax striatellus: conserved genome rearrangement in Delphacidae and discovery of new characteristics of atp8 and tRNA genes. BMC Genomics. 2013; 14:417.

19. Mashhoor K, Akhilesh VP, Sebastian CD, Rosy PA and Kottickal LV. Molecular Phylogenetic Status of Microcentrum rhombifolium in the Family Tettigoniidae, Developmental Microbiology and Molecular Biology. 2012; 3: 9-15

20. Nei M. Molecular Evolutionary Genetics, Columbia University Press, New York New Delhi. 1987

21. Irwin DM., Kocher TD and Wilson AC. Evolution of the cytochrome b gene of mammals. J. Mol. Evol. 1991; 32:128-44.

22. Schuh RT, Slater JA. True bugs of the world Hemiptera: Heteroptera: classification and natural history. New York, the United States of America: Cornell University Press. 1995.

23. Song $\mathrm{N}$ and Liang AP. Complete mitochondrial genome of the small brown planthopper, Laodelphax striatellus Delphacidae: Hemiptera, with a novel gene order. Zool Sci. 2009; 26:851-860.

24. Cryan JR.and Urban JM. Higher level phylogeny of the insect order Hemiptera: is Auchenorrhyncha really paraphyletic? Syst Entomol. 2012; 37:7

25. Song N, Liang AP and Ma C. The complete mitochondrial genome sequence of the planthopper, Sivaloka damnosus. J Insect Sci. 2010; 10:76.17.

\section{How to cite this article:}

Mashhoor K., Lazar K.V., Shanas S. and Ramesh N. Genetic Structure and Phylogenetic Status of Rice Brown Plant Hopper (BPH), Nilaparvata lugens Isolated from Kerala, India. J App Biol Biotech. 2015; 3 (05): 015-018. DOI: 10.7324/JABB.2015.3503 